\title{
Toward the Analysis of JWST Exoplanet Spectra: the effective temperature in the context of direct imaging
}

\author{
Jean-Loup Baudino, ${ }^{\star}$ J. Taylor, ${ }^{1}$ P. G. J. Irwin ${ }^{1}$ and R. Garland ${ }^{1}$ \\ ${ }^{1}$ Department of Physics, University of Oxford, Oxford OX1 3PU, UK
}

Accepted 2019 September 28. Received 2019 September 27; in original form 2019 July 9

\begin{abstract}
The current sparse wavelength range coverage of exoplanet direct imaging observations, and the fact that models are defined using a finite wavelength range, lead both to uncertainties on effective temperature determination. We study these effects using black-bodies and atmospheric models and we detail how to infer this parameter. Through highlighting the key wavelength coverage that allows for a more accurate representation of the effective temperature, our analysis can be used to mitigate or manage extra uncertainties being added in the analysis from the models. We find that the wavelength range coverage will soon no longer be a problem. An effective temperature computed by integrating the spectroscopic observations of the James Webb Space Telescope (JWST) will give uncertainties similar to, or better than, the current state-of-the-art, which is to fit models to data. Accurately calculating the effective temperature will help to improve current modelling approaches. Obtaining an independent and precise estimation of this crucial parameter will help the benchmarking process to identify the best practice to model exoplanet atmospheres.
\end{abstract}

Key words: planets and satellites: fundamental parameter — planets and satellites: gaseous planet — radiative transfer

\section{INTRODUCTION}

Since the first confirmation of a directly detected exoplanet by Chauvin et al. (2005), the effective temperature $\left(T_{\text {eff }}\right)$ has been one of the main parameters analysed. Exoplanet direct imaging gives planetary astronomers the opportunity to characterise the atmospheres of young giant exoplanets using their emission spectra obtained with ground-based instruments such as the Spectro-Polarimetric High-contrast Exoplanet REsearch (SPHERE Beuzit et al. 2008, Beuzit et al. 2019), the Gemini Planet Imager (GPI Macintosh et al. 2014) or soon from space with the Near-Infrared Spectrograph (NIRSpec) and the Mid-Infrared Instrument (MIRI, Rieke et al. 2015, Wright et al. 2015) of the JWST. The current state-of-the-art of instrumentation probes the outer part ( $\geq 10 \mathrm{AU}$ ) of young (<100 Myrs) stellar systems, studying young exoplanets with spectra overly dominated by selfemission coming from formation and contraction processes.

The temperature of an astrophysical object can be defined by the effective temperature (Hopkins 1864), it is theoretically defined by using the black-body theory (Violle 1842 Le Chatelier 1842, Wilson \& Gray 1894):

$M=\int_{0}^{+\infty} F_{\lambda} d \lambda=\sigma T_{\mathrm{eff}}^{4}$

^ E-mail: dr.jean-loup.baudino@hotmail.com where $M$ is the bolometric flux per unit surface area, $F_{\lambda}$ is the emission flux density in $W^{-2} \mu m^{-1}$, and $\sigma$ the Stefan-Boltzmann constant.

There are three techniques that are used to determine the effective temperature of an exoplanet. Generally the community fits the data with models (e.g. Bonnefoy et al. 2016), mainly to deal with the sparse wavelength coverage of real observations, for the majority of the exoplanets, in the Near Infrared (NIR, between $J$ and $L$ bands, i.e. $\sim 1.2$ $4 \mu \mathrm{m})$. The models used are also defined on a finite wavelength range, so that the integration is defined over a limited wavelength range and not between 0 and $+\infty \mu \mathrm{m}$ as in the Eq. 1. This situation can introduce an intrinsic error on the estimated $T_{\text {eff }}$.

Retrieval approaches struggle to determine $T_{\text {eff }}$, intrinsically because this technique computes the spectrum of the atmosphere only where the data is available and instead will often focus on retrieving a temperature profile that can be difficult to link to $T_{\text {eff }}$.

Another approach for estimating $T_{\text {eff }}$ was proposed by Morzinski et al. (2015), who extrapolated directly the bolometric flux from the data for the planet $\beta$ Pictoris $\mathrm{b}$ (assuming black-body emission spectra). As we show later in this paper, this approach was successful thanks to an exceptional data quality and coverage, and 
we will look ahead to what we will be able to do with JWST.

The JWST is the next generation of space-based international observatory. It is planned to be launched in early 2021, and will be game-changing in terms of exoplanet characterisation, especially for directly-imaged exoplanets (Danielski et al. 2018). As soon as the observations begin, by combining observations from two instruments (NIRSpec and MIRI) with a resolution and signal-to-noise ratio (SNR) never obtained before, the community (Early Release Science programme ID 1386, PI Sasha Hinkley) plans to obtain the first complete measurement of the spectral energy distribution (SED) of an exoplanet. With a SNR $>100$, the spectra provided by JWST will have better quality than the current state-of-the-art of modelling (Baudino et al. 2017) and will cover the major part of the emission flux of the planets.

The this paper give a review of the current technique fo inferring $T_{\text {eff }}$ from observations to explore the weaknesses and help modellers manage the intrinsic errors linked to these methods. We then highlight and quantify one of the opportunities given to the community by the JWST to obtain a rigorous estimate of $T_{\text {eff }}$ directly from the observations and the impact of this on the future of exoplanet atmosphere modelling.

We begin this paper by exploring the effect of wavelength range completeness (Sec. 2) using simple black-body functions and synthetic spectra from Exo-REM (a radiativeconvective atmospheric model of exoplanets Baudino et al. 2015, 2017) and two other models from the literature (Ydwarf model from Morley et al. 2012 and DriftPHOENIX, Helling et al. 2008). We follow by reviewing how the effective temperature is inferred, including a proposal of how it can be achieved using retrieval methods and a way to evaluate this parameter without models (Sec. 3). We then quantify the uncertainties on the temperature using the future observations of the JWST (Sec. 4) and we explain how this may be used to improve exoplanet modelling. Finally we summarise our conclusions (Sec. 5 ).

\section{COMPLETENESS EFFECT}

In this section, we explore in depth the calculation of $T_{\text {eff }}$. First, we compare pure black-body spectra to more realistic spectra generated by self-consistent models with various wavelength range coverages. We also show that we can use black-body spectra as a proxy for models.

\subsection{Black-body}

Our first approach is to explore the effect of estimating $T_{\text {eff }}$ from an incomplete wavelength range for the case of simple black-body emission. We use black-body spectra for a range of $T_{\text {eff }}$ between 400 and $1800 \mathrm{~K}$ with step size of $200 \mathrm{~K}$; for each case we integrate the spectrum between a minimum of $0.3 \mu \mathrm{m}$ and a maximum $\lambda_{c u t}$ between 5 and $500 \mu \mathrm{m}$ in $M\left(T_{\mathrm{eff}}, \lambda_{\text {cut }}\right)$.

Then we study how $M\left(\left(T_{\mathrm{eff}}, \lambda_{\text {cut }}\right) / \sigma\right)^{1 / 4}$ diverges com- pared to $M\left(\left(T_{\mathrm{eff}}, 500 \mu m\right) / \sigma\right)^{1 / 4}$ using the resultant absolute difference in temperature $\delta T$ :

$\delta T=T_{\mathrm{eff}} * \frac{M\left(\left(T_{\mathrm{eff}}, \lambda_{c u t}\right) / \sigma\right)^{1 / 4}-M\left(\left(T_{\mathrm{eff}}, 500 \mu m\right) / \sigma\right)^{1 / 4}}{M\left(\left(T_{\mathrm{eff}}, 500 \mu m\right) / \sigma\right)^{1 / 4}}$

We approximate the $\delta T$ as an intrinsic uncertainty of the $T_{\text {eff }}$ resulting from limiting the wavelength range.

We also performed tests on the minimum $(0.3 \mu \mathrm{m})$ and maximum $(500 \mu \mathrm{m})$ wavelength boundaries and expanding the wavelength range (for example from $0.1-1000 \mu \mathrm{m}$ ) did not significantly impact our results.

\section{$2.2 \quad$ Atmospheric models}

To test our analysis on more realistic cases, we make use of six models spanning the temperature range. With Exo-REM we generate two test cases with $T_{\text {eff }}=500$ and $1500 \mathrm{~K}$. We also complete the comparison by selecting two models from the literature with two different $T_{\text {eff }}: 200 \mathrm{~K}$ and $450 \mathrm{~K}$ for the Ydwarf model and $1000 \mathrm{~K}$ and $1800 \mathrm{~K}$ for Drift-PHOENIX. Following the method described in Sec. 2.1 we compare our black-body cases with these test cases. The data, except for Exo-REM, was taken from the VOSA Theoretical spectra web server 1 (Bayo et al. 2008).

\subsection{Theoretical effect}

To begin the analysis, we first compare the relationship between the uncertainties on the final estimated $T_{\text {eff }}$ and the completeness of wavelength coverage of the model used ("model" is used here as a generic word for either black-body or more complex models). Fig. 1 shows the uncertainties on the $T_{\text {eff }}, \delta T$, compared to the $\lambda_{c u t}$ for all the black-body spectra and the model test cases with a colour code linked to the temperature.

The first visible effect is the fact that the lower $T_{\text {eff }}$ will be mostly significantly impacted by the wavelength range completeness. This is easy to explain by the fact that the maximum of emission is, by definition, more in the red part (toward the longer wavelengths) of the spectrum for the cold cases, so with a maximum near $\lambda_{c u t}$ and consequently with more flux missing.

Secondly the selection of the $\lambda_{\text {cut }}$ impacts the $\delta T$ monotonically and logarithmically due to the fact that the maximum of the flux is always included in the wavelength range $(\sim 7 \mu \mathrm{m}$ for $400 \mathrm{~K})$ and we are just completing the integration with the remaining part of the tail of the blackbody function. For the worst case shown $\left(T_{\mathrm{eff}}=400 \mathrm{~K}\right)$ a $\lambda_{\text {cut }}>70 \mu \mathrm{m}$ gives a $\delta T<1 \mathrm{~K}$.

If we focus on non-negligible uncertainties $(\delta T>1 \mathrm{~K})$, model cases are similar or better in $\delta T$ compared to their black-body analogues. This effect can be explained by the fact that, generally, models generate spectra with the vast majority of the energy concentrated below $10 \mu \mathrm{m}$. Hence, for the next section we can use the black-body approximation for the shape of the spectra, this is representative of the worst case scenario.

\footnotetext{
1 http://svo2.cab.inta-csic.es/theory/newov2/
} 


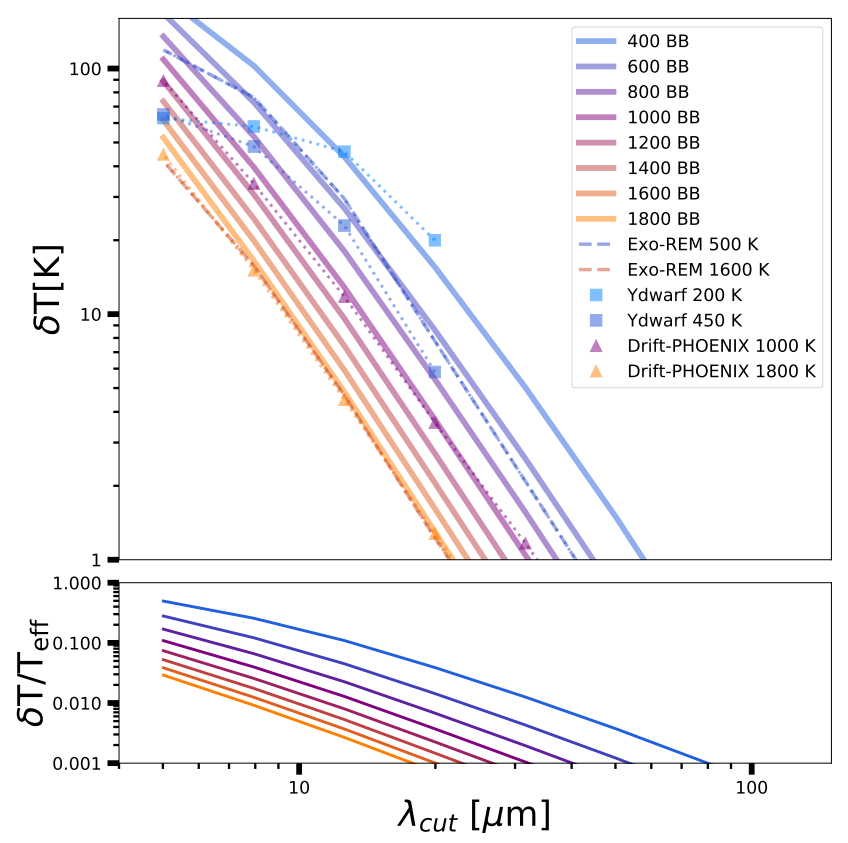

Figure 1. Top panel: The differences $(\delta \mathrm{T})$ between the $T_{\text {eff }}$ calculated from $0.3 \mu \mathrm{m}$ to $\lambda_{c u t}$, compared with an integration between 0.3 and $500 \mu \mathrm{m}$ (eq. 2), depending of the wavelength where the integration is stopped, $\lambda_{c u t}$. The colour code is linked to the temperature. Plain curves are the black-body emissions (BB). The dashed lines are for Exo-REM. The dotted lines with squares are for Ydwarf. The dotted with triangles are for Drift-PHOENIX. Bottom panel: Ratio between $\delta \mathrm{T}$ and $T_{\text {eff }}$ compared with $\lambda_{c u t}$. The color code is identical to the top panel.

\section{EVALUATION OF THE TEMPERATURE}

In this section we detail how observations can be used to infer the $T_{\text {eff }}$ using self-consistent models, retrieval or by directly using the data. Hence, there are three ways to infer $T_{\text {eff }}$.

\subsection{Self-consistent models}

For self-consistent models, the $T_{\text {eff }}$ is an input parameter used to generate a spectrum. The data are compared to a set of spectra: the subset of spectra that best reproduce the data give the $T_{\text {eff }}$ of the observed target (e.g. Macintosh et al. 2015, Bonnefoy et al. 2018). In this approach the uncertainty coming from the completeness of the wavelength range is not obvious to manage. For example in Exo-REM the spectrum is computed between 0.65 and $500 \mu \mathrm{m}$ : according to the previous section the impact is negligible in this case. But each model has its own definition and the Drift- PHOENIX and Ydwarf spectra are available in the VOSA database between 0.0001 and $950 \mu \mathrm{m}$ and 0.4 and $50 \mu \mathrm{m}$ respectively. This last range could be problematic if that was the range over which the structure of the atmosphere was computed, but this is only the published version of the spectra, as Morley et al. (2012) refers (Sec. 2.3) to a wavelength range of 0.268 to $227 \mu \mathrm{m}$ for the model computation itself.

The $T_{\text {eff }}$ and its relative uncertainty is normally derived by combining the results of various models from various teams. This is done to prevent the result being biased by the hypothesis of one model. The range of $T_{\text {eff }}$ will either be defined by the "best" result for each model (e.g. Bonnefoy et al. 2018) or by combining the range of models reproducing the data at a given level of uncertainty (e.g. Baudino et al. 2015). The "best" result is normally determined using the $\chi^{2}$ or the $G$ goodness-of-fit parameter (Cushing et al. 2008) and some models can be discarded in the process if they don't reach a given threshold. Instead of keeping an unique solution for each model it is also possible to keep the set of solutions for each model where the $\chi^{2}$ is linked to a given confidence interval (e.g. 1, 2, $3 \sigma$ ).

The typical uncertainty quoted in the literature is often around 100 to $200 \mathrm{~K}$ for an inferred temperature range between 500-2000 K (with many objects with a $T_{\text {eff }}$ around $1000 \mathrm{~K})$.

The main bias arising from self-consistent models comes from the physics that has been assumed, but self-consistent model results are also impacted by our knowledge in terms of theoretical spectroscopy (Baudino et al. 2017). However, following the improvement of the data quality (increased signal-to-noise ratio, resolution, wavelength coverage), we can increase the complexity our models, hence the number of free parameters. One convenient method to use in this case, coming from Earth and Solar System studies, is to use a retrieval approach (e.g. Irwin et al.|2008) that is not biased by the physics considered.

\subsection{Retrieval}

Defining the $T_{\text {eff }}$ using a retrieval technique is more difficult. Driven by the observations, retrieval techniques intrinsically do not generate the full spectrum and, by doing so, cannot determine this parameter.

The temperature is taken into account by retrieving the temperature profile where often one parameter can be similar to the $T_{\text {eff }}$ (e.g. $T_{\text {int }}$ in Lavie et al. 2017, is similar for three of the four planets of the HR 8799 system), but this parameter is difficult to compare with self-consistent models. The other possibility is to consider a given atmosphere (e.g. grey) and so the $T_{\text {eff }}$ can be derived as a simple parameter of the temperature profile (e.g. Garland 2018 , sec. 6.2.1). However, this last approach leads to a difference in the $T_{\text {eff }}$ compared to self-consistent models; for example, we observed a difference of 200-300 K higher, by applying for GJ 504 b (test done to prepare Bonnefoy et al. 2018) the profile defined in Garland (2018).

If the observations are good enough and include a wavelength range with molecular features, another approach that we are proposing is to retrieve at least the temperature profile and molecular abundances of molecules with global impact (mainly $\mathrm{H}_{2} \mathrm{O}$ ). We can then use this result to define an atmospheric structure and generate a full spectrum of the object that can be used to compute $T_{\text {eff }}$. This last approach does not retrieve the parameter directly, but gives the temperature for the retrieved atmosphere and gives similar results compared to self-consistent models. This will be explored in a later study. 
Even though retrievals are "data-driven", there are still potential biases arising from model assumptions, similar to those of self-consistent models, such as how the radiative transfer calculation is actually computed (e.g. assumptions of line shape, sub-Lorentzian correction, etc.). In the next section we show how we can try to avoid that, by using directly the observational data to compute the $T_{\text {eff }}$.

\subsection{Extrapolation from the observation}

Occasionally, the observational data cover a sufficient wavelength range to enable us to attempt to derive $T_{\text {eff }}$ by extrapolating it from the observation (Morzinski et al. 2015). $\beta$ Pictoris $b$, the planet studied by Morzinski et al. (2015), is one of the most studied directly-imaged objects and the large wavelength coverage available $(0.9-4.5 \mu \mathrm{m})$ is pretty unique. By integrating the observed flux and completing it by a black-body approximation outside the observed wavelength range to cover 0.001 to $100 \mu \mathrm{m}$, and by evaluating the error bars using a Monte Carlo experiment (Sec. 4.4 in Morzinski et al. 2015), this study finds a $T_{\text {eff }}$ consistent with the comparison of the data (Tab. 15 in Morzinski et al.|2015) with self-consistent models and shows a first step towards an independent determination of this temperature.

The biggest problem with applying this method is the current need to combine various instruments (e.g. NaCo, MagAO and GPI) from various observatories (e.g. Very Large Telescope, Magellan, Gemini South) that are not well cross-calibrated and with the flux extracted using various techniques (e.g. Angular Differential Imaging, Principal Component Analysis, Spectral Deconvolution) to populate the wavelength range.

It is within this context that we show, in the following section, how JWST will change the way how we derive $T_{\text {eff }}$.

\section{USING OBSERVATIONS OF THE JWST}

In this section we analyse the uncertainties on $T_{\text {eff }}$ if this parameter is defined observationally using JWST spectroscopy, without taking into-account the uncertainty on the radius. Fig 2 (black crosses) shows the error on $T_{\text {eff }}$ using the JWST NIRSpec and MIRI spectral capabilities, i.e. with a spectrum covering $0.6-13 \mu \mathrm{m}$. The uncertainties derived directly are expected to be low, $(<10 \%$ for the worst case). This gives us the opportunity to obtain $T_{\text {eff }}$ without modelling biases and with an accuracy greater than the current state-of-art using models illustrated in the figure of, respectively: 51 Eridani b (Rajan et al. 2017), VHS 1256-1257 b (Gauza et al. 2015), HIP 65426 b (Chauvin et al. 2017) and $\beta$ Pictoris b (Chilcote et al. 2017).

Using the SNR expected for VHS 1256-1257 b (i.e. observation of half an hour, with Prism and LRS mode, see Baudino et al. 2017), we also generated the error on $T_{\text {eff }}$ taking into account favorable, but realistic uncertainties in Fig.2 (red triangles), without taking into-account the fact that the radius is unknown.

We observe two regimes. First, lower than $800 \mathrm{~K}$, there is no visible difference with the cases with and without observational uncertainties. This comes from the fact that the

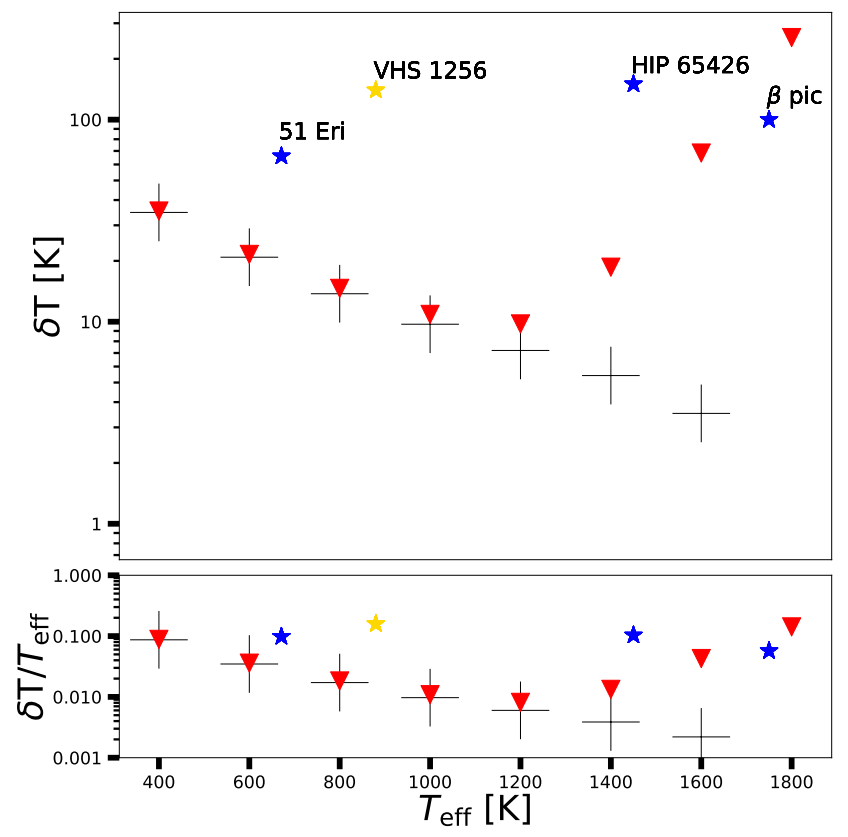

Figure 2. Top panel: The uncertainties $(\delta \mathrm{T})$ relative to the $T_{\text {eff }}$ calculated for a black-body spectra covering only the NIRSpec and MIRI wavelength ranges (black crosses and red triangles), without the consideration of radius uncertainty. The black crosses take into account only the effect of wavelength range completeness. The red triangles additionally take into account the uncertainties expected for VHS 1256-1257 b (expected to be one of the best targets). For the comparison with the current state-ofthe-art, the stars indicate the estimated uncertainties of $T_{\text {eff }}$ for four observed exoplanets, already published, with ground-based observations: 51 Eridani b, VHS 1256-1257 b, HIP 65426 b and $\beta$ Pictoris $\mathrm{b}$, characterised using comparison with models. Bottom panel: Ratio between $\delta \mathrm{T}$ and $T_{\text {eff }}$ relative to the $T_{\text {eff }}$.

error is dominated by the lack of data because the maximum of the flux is near the maximum of MIRI LRS and so we lose a lot of flux.

Then, comparatively, for $T_{\text {eff }}>800 \mathrm{~K}$ we observe an increase of the $\delta \mathrm{T}$. This comes from the fact that the maximum of the flux is shifted toward the visible part of the spectrum, where NIRSpec has a poorer SNR. This, combined with the fact that the flux is high, i.e. the temperature is high, leads to $\delta$ T exceeding $10 \mathrm{~K}$.

This picture is not complete because the true data give the observed spectrum, that will be linked to the $F_{\lambda}$ in Eq. 1 through a "dilution factor" $\frac{R^{2}}{D^{2}}$ where $R$ is the radius of the planet and $D$ the distance between the planet and the observer. The radius is not known but can be inferred. One possibility is to use the spectra and evolution models combined with the aged of the systems as shown in Males et al. (2014) and Morzinski et al. (2015). This will give an estimation of the radius, mass and $T_{\text {eff }}$. Using sparse ground-based data, Morzinski et al. (2015) found errors around $20 \mathrm{~K}$ and $0.02 R_{\text {Jup }}$. Even by using a more conservative error, we should be able to obtain similar or better result using JWST.

The $T_{\text {eff }}$ will be obtained directly by JWST spectroscopy with a good uncertainty, probably similar or better than 
the current uncertainties derived from ground-based observations compared with models (stars in Fig. 2).

Being able to achieve this precision without using models will be crucial to obtain benchmark cases to improve modelling (self-consistent and retrieval). As the community is currently trying to deal with many unknowns to generate accurate spectra (Baudino et al. 2017) especially for young (or highly irradiated) objects, being able to fix one important parameter such as the $T_{\text {eff }}$ will narrow the range of acceptable solutions to a more manageable set. If the differences between models (such as alkali and molecular far wing lineshape) are known, it will enable us to identify the best hypothesis in these models. This work will need to be done before any precise analysis of the JWST observation can be made and the direct-imaging ERS proposal assesses this properly as VHS 1256-1257 b will be observed using the modes used previously in this paper.

\section{CONCLUSIONS}

We performed an analysis of the uncertainty of the estimated $T_{\text {eff }}$ of exoplanets depending on the observed/defined wavelength range and we quantified the link between using a given wavelength range and the inferred $T_{\text {eff }}$. This can be used to optimise the modelling of exoplanet atmospheres. Computing the spectrum over a large wavelength range is time consuming so modellers need to choose a maximum wavelength. We have shown here that a maximum wavelength greater than $70 \mu \mathrm{m}$ is sufficient to have an intrinsic error on the $\delta T_{\text {eff }}<1 \mathrm{~K}$, for planets with an $T_{\text {eff }}$ in the range 400-1800 K. If models use a shorter wavelength range then Fig. 1 can help to impose a modelling uncertainty on the $T_{\text {eff }}$.

In cases where we will be able to obtain the full spectrum combining NIRSpec and MIRI we shown that we will obtain directly a good (similar or better than what we can currently achieve with SPHERE or GPI) characterisation of the $T_{\text {eff }}$ (i.e. with $\delta \mathrm{T}<100 \mathrm{~K}$ ). This kind of characterisation will be crucial to benchmark and update our models and approaches to analyse exoplanet direct-imaging observations. In the context of the JWST it will be mandatory to take into account the intrinsic modelling errors, as shown in this paper, for the $T_{\text {eff }}$.

Obtaining an accurate independent evaluation of $T_{\text {eff }}$ will lead us to improve our models (self-consistent and retrieval). To succeed to do so properly we will need to have a clear view of the differences and similarities between the models available in the literature. One of the methods to do so is to continue the benchmark process initiated by Baudino et al. (2017).

\section{ACKNOWLEDGEMENTS}

J.L.B. acknowledges the support of the UK Science and Technology Facilities Council. This publication makes use of VOSA, developed under the Spanish Virtual Observatory project supported from the Spanish MICINN through grant AyA2011-24052. This research made use of Numpy: Oliphant (2006), Van Der Walt et al. (2011). This research made use of Astropy, ${ }^{2}$ a community-developed core Python package for Astronomy (Astropy Collaboration et al. 2013. 2018). The authors thank the referee for the useful comments.

\section{REFERENCES}

Astropy Collaboration et al., 2013, A\&A 558, A33

Astropy Collaboration et al., 2018, AJ. 156, 123

Baudino J.-L., Bézard B., Boccaletti A., Bonnefoy M., Lagrange A.-M., Galicher R., 2015, Astronomy \& Astrophysics, 582, A 83

Baudino J.-L., Mollière P., Venot O., Tremblin P., Bézard B., Lagage P.-O., 2017, The Astrophysical Journal, 850, 150

Bayo A., Rodrigo C., y Navascués D. B., Solano E., Gutiérrez R., Morales-Calderón M., Allard F., 2008, Astronomy \& Astrophysics, 492, 277

Beuzit J.-L., et al., 2008, SPIE

Beuzit J. L., et al., 2019, submitted to A\&A, p. arXiv:1902.04080

Bonnefoy M., et al., 2016, Astronomy \& Astrophysics 587, A58

Bonnefoy M., et al., 2018, A\&A 618, A63

Chauvin G., Lagrange A.-M., Dumas C., Zuckerman B., Mouillet D., Song I., Beuzit J.-L., Lowrance P., 2005, Astronomy \& Astrophysics 438, L25

Chauvin G., et al., 2017, Astronomy \& Astrophysics 605, L9

Chilcote J., et al., 2017, The Astronomical Journal 153, 182

Cushing M. C., et al., 2008, ApJ 678, 1372

Danielski C., Baudino J.-L., Lagage P.-O., Boccaletti A., Gastaud R., Coulais A., Bézard B., 2018, AJ, 156, 276

Garland R., 2018, PhD thesis, University of Oxford, $\quad$ https://ora.ox.ac.uk/objects/uuid: 9f965479-bccc-4cad-8c6c-715368b89dca

Gauza B., Béjar V. J. S., Pérez-Garrido A., Osorio M. R. Z., Lodieu N., Rebolo R., Pallé E., Nowak G., 2015, The Astrophysical Journal, 804, 96

Helling C., Dehn M., Woitke P., Hauschildt P. H., 2008, The Astrophysical Journal 675, L105

Hopkins W., 1864, Transactions of the Cambridge Philosophical Society, 9, 628

Irwin P., et al., 2008, Journal of Quantitative Spectroscopy and Radiative Transfer 109,1136

Lavie B., et al., 2017, AJ, 154, 91

Le Chatelier H., 1842, Comptes rendus hebdomadaires des séances de lâĂŹAcadémie des sciences, publiées par MM. les secrétaires perpetuels, commencant au 3 août 1835, 114, 737

Macintosh B., et al., 2014, Proceedings of the National Academy of Sciences 111, 12661

Macintosh B., et al., 2015, Science 350, 64

Males J. R., et al., 2014, ApJ 786, 32

Morley C. V., Fortney J. J., Marley M. S., Visscher C., Saumon D., Leggett S. K., 2012, The Astrophysical Journal 756, 172

Morzinski K. M., et al., 2015, The Astrophysical Journal, 815, 108

Oliphant T. E., 2006, A guide to NumPy. Vol. 1, Trelgol Publishing USA

Rajan A., et al., 2017, The Astronomical Journal, 154, 10

Rieke G. H., et al., 2015, Publications of the Astronomical Society of the Pacific 127, 665

Van Der Walt S., Colbert S. C., Varoquaux G., 2011, Computing in Science \& Engineering, 13, 22

Violle J., 1842, Comptes rendus hebdomadaires des séances de lâĂŹAcadémie des sciences, publiées par MM. les secrétaires perpetuels, commencant au 3 août 1835, 114, 734

2 http://www.astropy.org 
Wilson W. E., Gray P. L., 1894, Philosophical Transactions of the Royal Society of London Series A 185, 361

Wright G. S., et al., 2015, Publications of the Astronomical Society of the Pacific 127, 595

This paper has been typeset from a $\mathrm{TE}_{\mathrm{E}} \mathrm{X} / \mathrm{LATEX}$ file prepared by the author. 